\title{
Propuesta metodológica para la generación de productos turísticos a partir de la comunidad local
}

\section{Methodological proposal for the emergence of touristic projects of the local community}

Dr. Omar Ismael Ramírez Hernández es profesor de tiempo completo de la Universidad Autónoma del Estado de México, en el Centro Universitario UAEM Temascalepec (México) (omar.ramirez.hdez@hotmail.com) (https://orcid.org/0000-0002-08745627).

\section{Resumen}

Las nuevas exigencias sociales, económicas y ambientales hacen necesario el diseño de productos turísticos donde la comunidad local sea considerada como el pilar fundamental para su creación y desarrollo. En este sentido, el objetivo central de la presente investigación es elaborar una propuesta metodológica para el diseño de productos turísticos a partir de la participación y gestión de este actor. En este sentido, la revisión de diferentes propuestas destaca la necesidad de crear una metodología para desarrollar productos turísticos a través de la comunidad local, pues es ella quien resalta como un actor olvidado en la generación y gestión de los productos turísticos. De esta forma, y cubriendo este vacío, la propuesta metodológica se compone de ocho etapas: I) Sensibilización de la comunidad local, II) Contextualización del lugar, III) Diseño del producto turístico, IV) Identificación y análisis de la demanda, V) Decisión del precio, VI) Comercialización, VII) Prueba de Mercado y VIII) Puesta en marcha del producto turístico y seguimiento. Si bien, la generación de productos turísticos es una labor heterogénea, y depende de muchos elementos intrínsecos y extrínsecos; este documento pretende ser una guía general que ayude a la integración de los residentes del destino como gestores de la actividad turística.

\begin{abstract}
The new social, economic and environmental demands make necessary to design touristic projects where the local community is considered as the foundation for their creation and development. The main objective of this article is to elaborate a methodological proposal to design touristic projects based on the participation and management of this community. In this sense, the literature review is used to develop touristic projects through the local community, since it is the local community that stands out as a forgotten part in the generation and management of touristic projects. Thus, the methodological proposal consists of eight stages: I) Sensitization of the local community, II) Contextualization of the place, III) Design of the tourism product, IV) Identification and analysis of the demand, V) Decision of the price, VI) Commercialization, VII) Market Test and VIII) Implementation of the touristic project and follow-up. Although the generation of touristic projects is a heterogeneous task, and it depends on many intrinsic and extrinsic elements, this document aims to be a general guide which helps to integrate the residents of the destination as managers of tourism activity.
\end{abstract}

\section{Palabras clave I keywords}

Planificación, metodología, productos turísticos, comunidad local, actor, participación.

Planning, methodology, tourism products, local community, actor, participation.

Cómo citar: Ramírez Hernández, O.I. (2019). Propuesta metodológica para la generación de productos turísticos a partir de la comunidad local. Retos Revista de Ciencias de la Administración y Economía, 9(17), 127-143. https://doi.org/10.17163/ret.n17.2019.08 


\section{Introducción}

El turismo se ha posicionado como una de las actividades económicas más importantes a escala mundial; por ello, ha tomado relevancia la creación de productos turísticos que diversifiquen la oferta de atractivos en los destinos y, por ende, satisfagan las necesidades de los turistas. De esta forma, autores como Bote (1990), Boullón (1994), Acerenza (1998), Godfrey y Clarke (2000), Gunn (2002), Zamorano (2002), Ivars (2003) y hasta la propia Organización Mundial del Turismo (1999), se han dado a la tarea de generar modelos de planificación turística.

A pesar de lo anterior, los modelos de planificación y creación de productos turísticos aplicados a países en vías de desarrollo, siguen teniendo una tendencia a gestionar los recursos de una forma «tradicional», en la que prevalece un alcance macroeconómico dejando a un lado aspectos sociales y ambientales (Ivars, 2003). Asimismo, aun cuando la sostenibilidad se convirtió en el principio rector de la planificación turística, solo se ha cambiado la perspectiva hacia una económica-ambiental (Osorio, 2006).

De esta forma, son pocos los destinos turísticos que se abocan a integrar a la comunidad local, a pesar de que ésta es uno de los actores (sino es que el principal) que puede detonar el desarrollo local (Aref, Gill, \& Farshid, 2010; Sebele, 2010). Por ello, ha surgido el término "turismo comunitario" [Community based tourism], que de manera general hace referencia a la generación y gestión de productos turísticos por la misma comunidad o actores locales en los destinos.

Así, Beeton (2006) propone generar modelos de planificación y propuestas de gestión más inclusivos y efectivos a partir del turismo de base comunitaria. Por tal razón, el objetivo de este trabajo se aboca en proponer una metodología que integre a la comunidad local en la generación de productos turísticos, no solo como atractivo por los recursos naturales y culturales con los que cuenta, sino como un actor participativo que proponga, desarrolle y lleve a cabo dichos productos.

Para ello, este documento realiza una revisión de diferentes metodologías o propuestas de planificación de productos turísticos, retomando algunas generadas desde la academia, consultorías y entes gubernamentales, entre las que se evidencia una incipiente participación e integración de la comunidad local como un actor principal. Así, este trabajo es organizado en seis partes: 1) turismo comunitario, II) comunidad local y su integración en la generación de productos turísticos, III) producto turístico, IV) metodología seguida, V) análisis de las diferentes formas de realizar un producto turístico, y, por último, VI) la propuesta metodológica.

\subsection{Turismo comunitario}

De acuerdo con Goodwin \& Santilli (2009) y Beeton (2006), el «turismo comunitario» [community based tourism] empieza a emplearse a finales del siglo XX, y surge a partir de la necesidad de gestionar el turismo alternativo a través de una forma sustentable. De esta manera, esta gestión retoma de la sustentabilidad sus tres dimensiones principales: ambiental, económica y social (García, Figueiró, \& Silva, 2013).

A pesar de que se ha ahondado en el abordaje del turismo comunitario, la literatura concluye que éste es polisémico y muchas veces hace referencia a proyectos relacionados con las comunidades locales (Rodrigues, Corbari, Cioce, \& Juremma, 
2014). Asimismo, en torno a éste han surgido otros términos que dificultan su conceptualización, tal es el caso de «gestión/manejo comunitario» (Ávila, 2002; Mehedi, Mohammed, Nassani, \& Nurul, 2017), «empresas de base comunitaria» (Monyara \& Jones, 2007; Senyana \& Moren, 2011) o "turismo rural comunitario» (Montoya, 2013). Sin embargo, estos términos no dejan en claro sus alcances, implicaciones o sustento teórico, lo cual ha originado complicaciones para definirlo (Ernawati, Sudarmini, \& Sukmawati, 2018).

No obstante, desde la academia han surgido algunas propuestas para definir al «turismo comunitario». Una de ellas es la elaborada por Dernoi (1988, citado en Pearce, 1992), quien menciona que es un conjunto de servicios ofertados por la comunidad local, con el objetivo de establecer una interacción directa con visitantes, surgiendo un entendimiento mutuo entre anfitriones e invitados. Conviene subrayar que esta definición genera algunas lagunas; sin embargo, el autor puntualiza sobre un elemento medular, y es que la comunidad local es quien tiene que generar la oferta de servicios (alojamiento, comida, actividades recreativas, entre otros). Esto coincide con lo argumentado por Torres (2015, p. 70) quien menciona que "debe ser la misma comunidad quien gestione los recursos con que cuenta. Son ellos quienes le dan significados o re significan a estos, dentro de un proceso permanente en el cual están involucrados".

Asimismo, es posible encontrar algunas otras definiciones que resaltan elementos específicos, visualizando al turismo comunitario como una estrategia para valorizar elementos históricos (Sampaio, Alves, \& Falk, 2008; Hiwasaki, 2006); mientras que otras ponen énfasis en la interacción local-visitante y sus experiencias (Jamal \& Getz, 1995; Nyaupane, Morais, \& Dowler, 2006; Sampaio, Alves, \& Falk, 2008). Algunas más se enfocan en la forma de participación (activa y pasiva) de la comunidad local en la gestión de los recursos naturales y culturales (v.gr. Hiwasaki, 2006; Okazaki, 2008; López-Guzmán \& Sánchez, 2009; Sebele, 2010).

Si bien, es posible encontrar diferentes definiciones sobre «turismo comunitario», se pueden identificar carateristicas que lo singularizan:

- Existe interacción directa entre comunidad local y visitantes.

- Intercambio cultural y económico entre comunidad local y visitantes.

- Experiencias «auténticas» a partir de la vida cotidiana de los locales.

- La comunidad local es la principal beneficiada.

- Conservación y preservación del patrimonio cultural y natural.

- La comunidad es encargada de la gestión de los recursos y servicios turísticos.

- El turismo comunitario es posible «únicamente» a nivel local ${ }^{1}$.

- La comunidad local se ve influida por la actividad turística en su dimensión social, ambiental y cultural.

Estas características mencionadas ut supra contextualizan el turismo comunitario, el cual ha sido utilizado para legitimar la industria turística, otorgado empo-

1 Si bien, el turismo comunitario pretende ser una nueva postura para afrontar problemas sociales (pobreza, desigualdad, desarrollo, entre otros); su acción está vinculada al ámbito local (Cfr. Blackstock, 2005; Beeton, 2006). 
deramiento a las comunidades (Blackstock, 2005). En esta línea, Fernández (2011) argumenta que la vía para impulsar el proceso de empoderamiento colectivo en el turismo es la participación comunitaria de las personas que viven en el destino. Esto es la situación que singulariza al turismo comunitario, en el que la participación de la comunidad local es fundamental para llevar a cabo la actividad turística.

En este sentido, el turismo comunitario no se establece como una nueva tipología de turismo, sino una forma de planear y gestionar la actividad turística a través de los actores relacionados con éste, dando prioridad a la comunidad local.

\subsection{La comunidad y su participación en los productos turísticos}

De acuerdo con Singh, Timothy \& Dowling (2003) y Monterrubio (2009), es posible reconocer dos tendencias claras para definir a la comunidad. La primera de ellas es la perspectiva geográfica, la cual hace alusión al espacio donde converge un grupo de individuos que delimitan sus formas de vidas y actividades económicas. La segunda tendencia es la social y antropológica, en donde la comunidad va más allá del espacio geográfico y los habitantes, tomando en cuenta relaciones, costumbres, tradiciones, valores, entre otros.

En este marco, cabe resaltar que el término «comunidad» engloba elementos como el espacio geográfico, tradiciones, costumbres, creencias, idioma, entre otros. También es reconocida la capacidad de los integrantes de la comunidad para interactuar en los diferentes procesos sociales, económicos y culturales, y por ende en el turismo (Boyd \& Singh, 2003). Sin embargo, muchos de los modelos de desarrollo turístico predisponen el beneficio económico al social, ocasionando que la participación de las comunidades sea casi nula (Blackstock, 2005; Sánchez, 2009).

Ruhanen (2009) reafirma que la comunidad local no ha sido tomada en cuenta en la planeación de los destinos turísticos, a pesar de que la literatura sugiere la participación efectiva de ésta para el desarrollo del turismo. De hecho, cuando la comunidad está integrada en la toma de decisiones, en muchas ocasiones su intervención es limitada, pues únicamente son tomadas en cuenta las opiniones con respecto al turismo, o solo conocen los posibles beneficios económicos y algunos costos que trae consigo esta actividad económica (Blackstock, 2005). Por ello, Sánchez (2009) destaca en su investigación que, a pesar de la generación de empleos, para la comunidad local no hay otros beneficios sociales.

Por ello, se reconoce la importancia de incorporar a la comunidad local en la gestión y desarrollo de la actividad turística, con el fin de evitar situaciones de inconformidad, insatisfacción o indiferencia de los residentes. De esta forma, Boonratana (2010), mediante el análisis de la literatura, reconoce las ventajas de tomar en cuenta a la comunidad local para el desarrollo y gestión de los productos turísticos:

- Las comunidades conservan una forma de vida y cultura tradicional;

- El turismo en las comunidades se planifica, desarrolla y gestiona con su consentimiento;

- Las comunidades participan activamente en la planificación, desarrollo y gestión de las actividades turísticas; 
- El proceso de planificación, desarrollo y manejo del turismo ayuda a unificar, empoderar e infundir orgullo en las comunidades;

- Las comunidades están facultadas para planificar, desarrollar y gestionar el turismo complementario a su estilo de vida;

- Todos los residentes de las comunidades tienen las mismas oportunidades para obtener beneficios;

- Los ingresos generados son una fuente adicional o alternativa, o se utilizan para aliviar la pobreza;

- Parte del ingreso turístico se reserva para proyectos que colectivamente benefician a las comunidades;

- El turismo fomenta la preservación/conservación del patrimonio cultural y natural de las comunidades;

- Los actores del turismo son conscientes de los impactos negativos del turismo y cuentan con medidas para mitigarlos;

- Se informa a los visitantes sobre las normas sociales y culturales de las comunidades antes o después de su llegada, fomentando así un comportamiento responsable;

- Los intercambios entre los visitantes y las comunidades fomentan la tolerancia, la comprensión y el aprendizaje intercultural; y

- Los actores del turismo están obligados a evitar la mercantilización de rituales y ceremonias (especialmente sagradas y/o religiosas).

A pesar de las ventajas de involucrar a la comunidad local en la planeación y desarrollo de la actividad turística, la literatura reconoce que la población local comúnmente es un actor olvidado (v.gr. Noia, Avila, \& Cartibani, 2009; Waligo, Clarke, \& Hawkins, 2013; Jovicic, 2014; Luna , 2014); pues en la mayoría de los destinos ésta participa de forma incipiente o nula en cuanto a la toma de decisiones sobre el desarrollo turístico. También es reconocida su difícil incorporación, ya sea por obstáculos políticos (Jaime, Casas, \& Soler, 2011; Palomino, Gasca, \& López, 2016), indiferencia hacia la actividad (Ibañez, Ivanova, \& Amador, 2010; Teye, Sönmez, \& Sirakaya, 2002), así como la falta de habilidad de los gestores turísticos en concienciarlos y hacerlos partícipes (Ruiz, Hernández, Coca, Cantero, \& Del Campo, 2008).

\subsection{El producto turístico y sus características}

El producto turístico es definido desde varias perspectivas. Desde el punto de vista económico, es concebido como el flujo de efectivo presente en la venta de conducentes a facilitar los viajes (De Borja, Casanovas, \& Bosch, 2002). No obstante, otros autores generan definiciones que tratan de entender la totalidad del producto turístico. Crespi \& Planells (2006) argumentan, en esta línea, que el producto turístico está determinado por los bienes y servicios ofertados para los turistas.

Si bien, en la literatura se pueden encontrar un sinfín de definiciones sobre los productos turísticos, Ugarte (2007) profundiza en sus características, las cuales se enlistan a continuación: 
- Tangible e Intangible: los productos turísticos poseen componentes tangibles; por ejemplo: la cama de un hotel, las amenidades, etc. La parte intangible, son todas aquellas características que están basadas en expectativas como la motivación, uso o experiencia.

- Caducidad: no es posible guardar o almacenar éstos para disfrutarlos después.

- Agregabilidad y sustituibilidad: ningún componente del producto es indispensable, por lo que alguno puede ser sustituido o agregado.

- Heterogeneidad: ningún producto es igual a otro.

- Subjetividad: la opinión del cliente al momento del consumo será distinta.

- Individualidad: las experiencias que produce son individuales.

- Inmediatez: Su consumo es simultáneo a su fabricación real.

- Simultaneidad de producción y consumo: el producto se crea, produce y consume al mismo tiempo.

De acuerdo a lo anterior, las cualidades que poseen los productos turísticos los diferencian de otros que se originan en los demás sectores económicos. Así también el producto está integrado por elementos de orden sociológico y psicológico, debido a que la creación de éstos parte de satisfacer las necesidades o deseos humanos. Por ello, Villalva (2011) enlista algunos de éstos:

1. Atractivos: Son las cosas o lugares que motivan la visita del turista.

2. Accesos y facilidades: Son los medios para obtener el producto turístico.

3. Herencia cultural de un pueblo: Es todo aquello relacionado con la cultura de un grupo social en particular; lo cual también puede convertirse en la principal motivación para los visitantes.

El primer y el último punto dan oportunidad para reconocer a la comunidad local como un actor importante en la generación de productos turísticos (Monterrubio, 2009), pues es ella a quien pertenecen los atractivos y la herencia cultural. En este sentido, los productos turísticos deben ser considerados como una oportunidad de desarrollo y crecimiento, no solo económico sino también social, por lo que existe la necesidad de hacer participar a la comunidad local en los procesos de creación y desarrollo de productos turísticos.

\section{Materiales y método}

El objetivo central de esta investigación es realizar una propuesta metodología para la planeación de un producto turístico a partir de la comunidad local como actor principal. Para conseguir este fin, este trabajo se realizó en dos grandes fases: la primera comprendió la búsqueda, comparación y análisis de diferentes métodos para la creación de productos turísticos, los cuales fueron diseñados por académicos, sector público y privado; mientras que la segunda fase consistió en la elaboración de una nueva propuesta metodológica.

La primera fase constó internamente de dos etapas: la primera, centrada en la búsqueda de literatura científica en repositorios, bases de datos e indexaciones como 
Redalyc, SciELO, Sage Journals, Sciencedirect, entre otros. Mientras que la segunda exploró la web, para acceder a otros trabajos que propusieran metodologías para la elaboración de productos turísticos.

Para estudiar las propuestas se tomó como punto de partida la investigación de Machado y Hernández (2007), quienes realizaron un análisis comparativo de los procedimientos para un producto turístico, los cuales deben reunir ciertos requisitos, como son: objetivos, necesidades de los clientes, generación de ideas, concepto de producto, estrategias de marketing, análisis del negocio, desarrollo del producto, prueba de mercado y lanzamiento del producto. Al respecto, para esta investigación se postularon los siguientes elementos:

1. Objetivos: fin que persigue la creación del producto turístico.

2. Estudio de mercado: análisis de la demanda para el producto.

3. Estudio de la oferta: evaluación de los atractivos con los que cuenta la comunidad local.

4. Desarrollo del producto: generación de ideas y creación de la «idea central» del producto turístico.

5. Estrategias de marketing: acciones para la promoción y venta del producto.

6. Prueba de mercado: se realiza una prueba piloto antes de lanzar el producto turístico al mercado.

7. Lanzamiento del producto: actividades para lanzar el producto turístico al mercado.

8. Seguimiento: actividades para dar seguimiento por parte de los gestores.

9. Sensibilización de la comunidad local: dar a conocer a la comunidad en general los elementos positivos y negativos que trae consigo al turismo.

10. Mapeo de actores: identificación de actores para la gestión del producto.

11. Forma de participación de la comunidad local: tipo de participación de la comunidad local, la cual puede ser pasiva, activa o nula.

De esta forma, se realizó un estudio comparativo de diferentes modelos de planeación para productos turísticos, en el que se identificó si las propuestas contaban con los elementos anteriormente planteados. Cabe decir que, aunque muchas propuestas no contaban con el nombre específico del elemento a analizar, sí contaban con un apartado similar. A partir de las debilidades identificadas se realizó la propuesta metodológica.

\subsection{Revisión de propuestas metodológicas}

El diseño de productos turísticos es un trabajo artístico y original que conlleva la búsqueda de beneficios económicos, sociales y medioambientales (Machado \& Hernández, 2007). De esta manera, los procedimientos son variados y cuentan con diversas etapas, elementos, factores y objetivos; esto debido a los diferentes contextos que tienen las personas, instituciones u organizaciones que los crearon. 


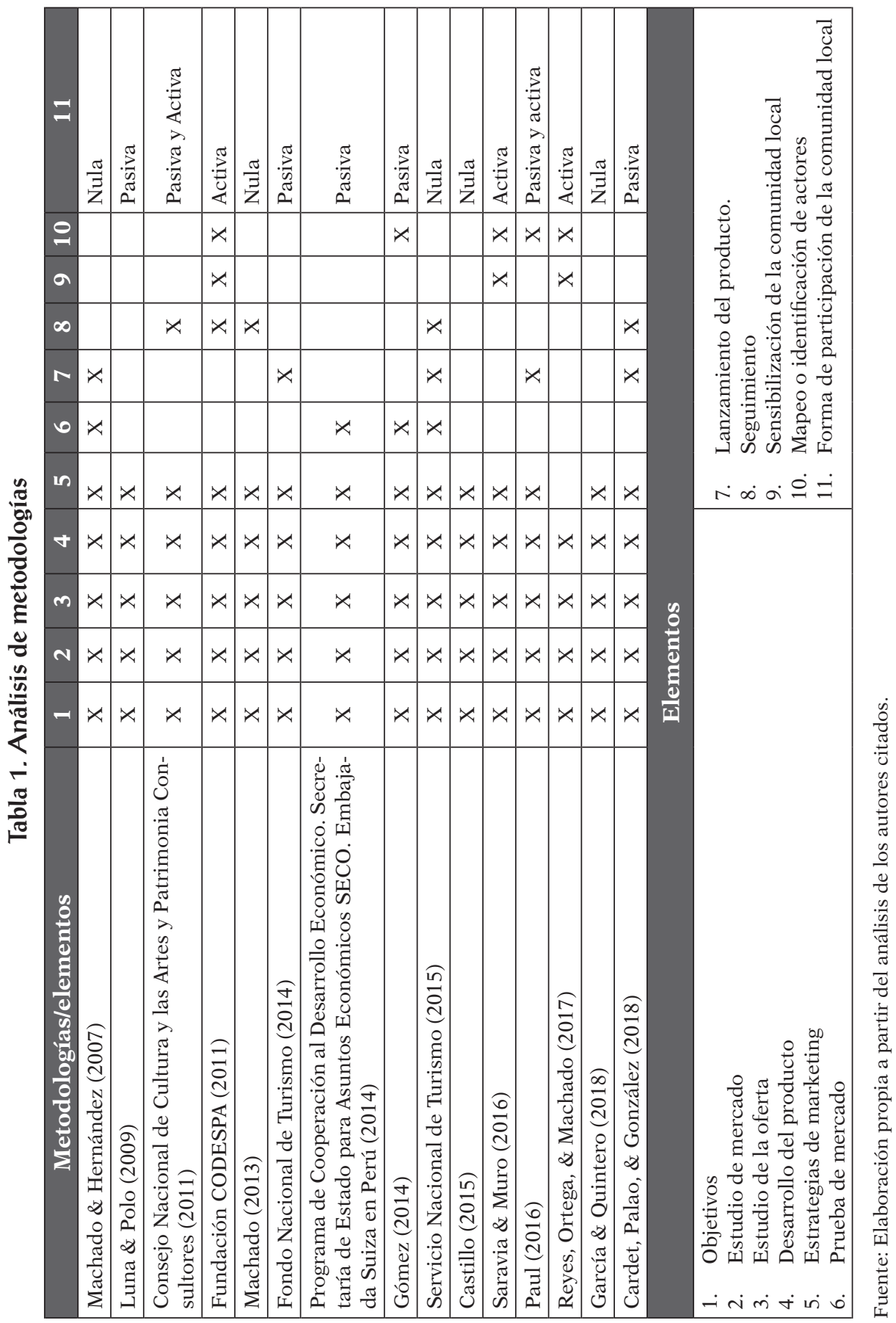


Del análisis crítico de la literatura se encontraron 15 propuestas para elaborar productos turísticos, en las que fue posible encontrar metodologías basadas únicamente en la oferta y demanda (v. gr. Machado \& Hernández, 2007; Luna \& Polo, 2009; Consejo Nacional de Cultura y las Artes y Patrimonia Consultores, 2011; Machado, 2013; Fondo Nacional de Turismo, 2014; Programa de Cooperación al Desarrollo Económico. Secretaría de Estado para Asuntos Económicos SECO. Embajada Suiza en Perú, 2014; Servicio Nacional de Turismo, 2015; Castillo, 2015; Cardet, Palao, \& González, 2018), mientras que, por otro lado, hay propuestas que apuestan a la inclusión de actores para detonar el desarrollo (v. gr. Fundación CODESPA, 2011; Gómez, 2014; Saravia \& Muro, 2016; Paul, 2016; Reyes, Ortega, \& Machado, 2017).

De manera general, la mayoría de las propuestas contemplan una participación nula o pasiva de la comunidad local, pues al estar basados en la oferta y demanda (enfoque económico), los actores locales solo son limitados a prestar servicios y no participan en la planeación de los productos, situación que no es benéfica para el desarrollo local (Reyes, Ortega, \& Machado, 2017). Adicionalmente, Saravia \& Muro (2016) mencionan que los productos turísticos deben ser validados por la comunidad.

En la misma postura, Mikery \& Pérez-Vázquez (2014, p. 1739) argumentan que "es necesario generar métodos de análisis más robustos e incluyentes que promuevan la gestión de productos turísticos; es decir, de enfoques integradores, participativos y en un proceso uso multifuncional". Así, resalta la inclusión este actor en la elaboración de productos turísticos mediante la participación activa, donde los locales generen las ideas y acompañen la gestión, el desarrollo, los productos y programas (ob. cit).

\section{Propuesta metodológica para la generación de productos turísticos a partir de la comunidad local}

Actualmente existe la creciente responsabilidad para crear productos turísticos que logren beneficiar a los diferentes actores del turismo, principalmente a la comunidad local. De esta forma, se propone la siguiente metodología, dividida en ocho etapas para la creación del producto turístico (ver figura 1). 
Figura 1. Metodología para la elaboración del producto

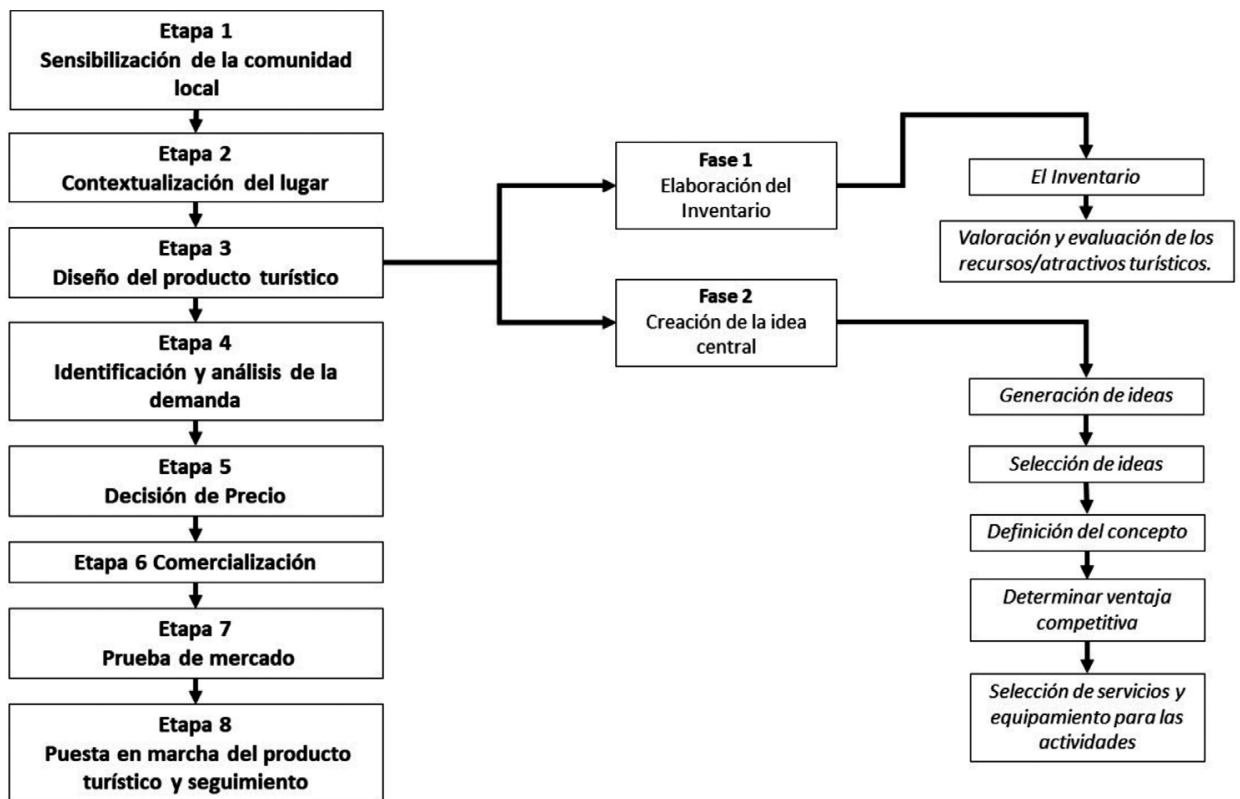

Fuente: Elaboración propia.

De acuerdo con la imagen anterior, se presentan las siguientes etapas: 1) Sensibilización de la comunidad local, 2) Contextualización del lugar, 3) Diseño del producto turístico, 4) Identificación y análisis de la demanda, 5) Decisión del precio, 6) Comercialización, 7) Prueba de mercado y 8) Puesta en marcha del producto turístico y seguimiento; asimismo, para llevar a cabo algunas de éstas, es necesario cumplir una serie de fases y pasos.

\subsection{Etapas previas}

\subsubsection{Etapa 1: Sensibilización de la comunidad local}

En esta etapa se llevan a cabo sesiones de sensibilización con la comunidad local, las cuales pretenden evaluar la aceptación sobre la creación del producto turístico, dando a conocer los beneficios y costos que el turismo puede traer a su comunidad. En caso de no ser favorable, se prescindirá del producto turístico asociado.

En caso de ser aceptada la propuesta, se procederá a identificar un equipo de trabajo. Este grupo deberá contener integrantes de cada uno de los actores encontrados en los destinos turísticos (sociales, culturales, económicos, públicos y privados). De acuerdo a Saravia \& Muro (2016), dichos actores dependerán de las características del destino. Asimismo, es importante reconocer que incorporar la participación y colaboración de cada actor relacionado con el turismo permitirá que ellos se sientan empoderados con el proyecto (Sebele, 2010). 


\subsubsection{Etapa 2: Contextualización del lugar}

Se realiza la caracterización del lugar donde se encontrará el nuevo producto turístico.

\subsubsection{Etapa 3: Diseño del producto turístico}

El proceso de diseño del producto abarca dos fases: elaboración del inventario turístico y la creación de la idea central, descritas a continuación. En este sentido, es importante que el grupo de trabajo conformado y la comunidad local evalúen los atractivos por igual, pues algunos autores (v. gr. Cardenas, 2006; Hernández, 2001; Machado \& Hernández, 2007; Villalva, 2011; Waligo, Clarke, \& Hawkins, 2013) opinan que los recursos turísticos deben constituirse en atractivos turísticos mediante un proceso técnico y diferentes puntos de vista.

Para dar cumplimiento a estos pasos es recomendable utilizar la técnica de investigación de grupos focales [focus groups], la cual es una entrevista a más de una persona, en donde abordan un tema predeterminado en presencia de un moderador (Peterson \& Barron, 2007; Bryman, 2012). Así, la comunidad local participará en la definición del producto turístico y los emprendimientos serán propiedad intelectual de la comunidad.

\subsection{Fases de planificación}

\subsubsection{Fase 1: Elaboración del inventario Turístico y evaluación}

Esta fase estará subdividida en dos: la primera comprende la elaboración del inventario turístico, mientras que la segunda hace alusión a la evaluación de los diferentes recursos que se encuentran en el sitio, para el posterior aprovechamiento de estos.

El inventario turístico

Un inventario turístico permite la selección de los atractivos disponibles de un territorio e identifica los lugares de interés turístico, para posteriormente, elegir aquellos que se consideren prioritarios, en los cuales se deben concentrar proyectos (Bote, 1990). De esta forma, para poder identificar los atractivos turísticos se sugiere utilizar el criterio de Hernández (2001), quien los clasifica en dos:

- Atractivos Naturales: son elementos creados por la naturaleza.

- Atractivos Culturales: son las manifestaciones humanas que tienen relación con la cultura (tradiciones, costumbres, artesanías, entre otras).

Valoración y evaluación de los recursos/atractivos turísticos

Para el producto turístico es necesario calificar los atractivos, lo cual debe hacerse a partir de sus características, y deberá responder a cuatro elementos básicos:

- Calidad.- Es el valor intangible y subjetivo inherente a sí mismo, centrado en la originalidad de los atractivos, y genera interés en la visita.

- Accesibilidad.- Son las condiciones físicas para llegar a los atractivos o lugares de interés. 
- Seguridad.- Son aquellas características operativas que hacen funcional al atractivo.

- Utilización para la actividad turística.- Es la viabilidad del atractivo para albergar la llegada de turistas.

\subsubsection{Fase 2: Creación de la idea central}

En esta fase se tratará de conceptualizar la idea de lo que se quiere en el producto. Se divide en siete pasos:

- Generación de ideas: La comunidad local aporta ideas sobre las actividades turísticas que pueden ser desarrolladas.

- Selección de ideas: elegir ideas que cumplan con los criterios de viabilidad (social, ambiental, cultural y económica).

- Definición del concepto: la comunidad local conceptualiza y contextualiza las actividades a desarrollar para el producto, para definir su tipo.

- Determinar ventaja competitiva: la comunidad aporta ideas para poder distinguir el producto de otros similares.

- Selección de servicios y equipamiento para las actividades: Se identificarán qué elementos son necesarios para el desarrollo del producto entre ellos el capital humano, los recursos materiales, económicos, normativos, entre otros.

\subsection{Fases de implantación}

\subsubsection{Etapa 4: Identificación y Análisis de la demanda}

El objetivo de esta etapa es identificar y analizar la posible demanda que pueda consumir el producto turístico. Para emprender un proyecto es necesario verificar si existe una oportunidad de mercado (Go \& Govers, 2000). A partir de ello, se debe realizar un análisis de la información referente a las tendencias actuales de los principales mercados emisores, su comportamiento, motivaciones, entre otros (Gómez, 2014).

\subsubsection{Etapa 5: Decisión de Precio}

El precio se fijará mediante el rango de ganancia el cual se quiera obtener, donde se toma en cuenta el costo de producción y la cantidad que podrían pagar los consumidores.

\subsubsection{Etapa 6: Comercialización}

En esta etapa son elegidos los canales de distribución del producto con base al resultado obtenido en la etapa cuatro (3.3.1). Adicionalmente, se propone la creación de una «marca» que distinga al producto ofertado en el lugar, pues ésta puede ayudar a consolidar un producto turístico (Kotler, Haider, \& Rein, 1993).

\subsubsection{Etapa 7: Prueba de Mercado}

El objetivo de esta etapa, es someter a prueba el producto "ante un grupo de turistas pertenecientes al segmento de mercado seleccionado” (Gómez, 2014, p. 165), lo 
cual consiste en ajustar los itinerarios, pudiendo reconsiderar los procesos de las actividades.

\subsubsection{Etapa 8: Puesta en marcha del producto turístico y seguimiento}

Esta etapa es la final de la elaboración y gestión del producto turístico, la cual tendrá una propuesta solida de actividades, servicios y beneficios a partir de una experiencia turística completa, lo que conlleva un producto turístico como tal (Machado \& Hernández, 2007). Asimismo, se tendrá que dar un seguimiento paulatino a las acciones llevadas a cabo, pues es posible implementar una mejora continua a partir de la revisión de los procesos, con el fin de consolidar, integrar y diversificar la oferta de servicios.

\section{Discusión y conclusiones}

Actualmente, la actividad turística es visualizada como un motor de desarrollo para las personas que habitan en los destinos. Sin embargo, se ha tratado de incluir la participación de la comunidad local en el turismo, con poco éxito (Ruhanen, 2009; Sebele, 2010). Por ello, resulta indispensable generar un proceso de planificación integrador, donde la comunidad local sea reconocida como pilar fundamental para la creación y gestión de los productos turísticos. En este sentido tiene su origen el turismo comunitario, no como una nueva tipología de turismo, sino como una manera de planeación y gestión de la actividad turística, basada en el paradigma de la sustentabilidad.

Si bien, en la actualidad existen propuestas para la planificación y generación de productos turísticos (v. gr. Bote, 1990; Boullón, 1994; Acerenza, 1998; Godfrey \& Clarke, 2000; Ivars, 2003; Saravia \& Muro, 2016), la literatura científica resalta la exclusión de la comunidad local en el proceso de planificación (v. gr. Ruhanen, 2009; Sebele, 2010; Waligo, Clarke, \& Hawkins, 2013; Saravia \& Muro, 2016), particularmente debido a situaciones políticas, indiferencia hacia la actividad o la poca labor de sensibilización realizada por los gestores turísticos. Por ello, esta investigación realizó una propuesta de una metodología integradora para la creación de un producto turístico, de tal manera que integre a la comunidad local de forma participativa.

A pesar de generar esta propuesta, una de las principales limitantes que se vislumbran en su aplicación son dos: la primera, centrada sobre la negativa o resistencia a la participación por parte de la comunidad local con la creación del producto turístico o del turismo en general. Cabe mencionar que esto depende de las habilidades de los gestores, pues en muchas de las ocasiones no cuentan con el poder de convencimiento para relacionarse con la comunidad local (Mair, 2012), lo que representa un reto a cubrir en futuras investigaciones.

La segunda limitante es que esta metodología no sirve para planificación de proyectos turísticos «macro», pues es centrada en grupos pequeños. De tal manera, Monterrubio (2009, p. 107) menciona que:

[...] la planificación a nivel local permite mayores oportunidades para el desarrollo exitoso del turismo; además de garantizar mejores condiciones de vida en las áreas social, cultural, económica y ecológica de la comunidad receptora. Es precisamente en este 
nivel donde la incorporación de la comunidad local juega un papel importante en la planificación y gestión exitosa del desarrollo turístico.

Por otro lado, la ventaja y diferencia de esta propuesta con otras metodologías está en el proceso de sensibilización de la comunidad local, pues en muchos de los casos este actor tiene poco conocimiento sobre lo que significa el turismo y los costos y beneficios que éste provoca (Aref, Gill, \& Farshid, 2010). Así, los integrantes de la comunidad son quienes proponen y definen el tipo de producto turístico a implementar, a partir del conocimiento que poseen sobre el espacio geográfico, tanto de sus recursos naturales y como los culturales.

\section{Referencias}

Acerenza, M. (1998). Administración del turismo, planificación y dirección. México: Trillas.

Aref, F., Gill, S., \& Farshid, A. (2010). Tourism development in local communities: as a community development approach. Journal of american Science, 6(2), 155-161. https://doi.org/10.7537/ marsjas060210.26

Ávila, V. (2002). Community-based ecotourism management moving towards sustainability, in Ventanilla, Oaxaca, Mexico. Ocean $\&$ Coastal Management, 45, 511-529. https://doi. org/10.1016/S0964-5691(02)00083-2

Beeton, S. (2006). Community development through tourism. Collingwood, Australia: Landlinks Press.

Blackstock, K. (2005). A critical look at community based tourism. Community development journal, 40(1), 39-49. https://doi.org/10.1093/cdj/bsi005

Boonratana, R. (2010). Community-based tourism in thailand: the need and justification for an operational definition. Kasetsart Journal Social Science, 31(2), 280-289.

Bote, V. (1990). Planificación económica del turismo. México: Trillas.

Boullón, R. (1994). Planificación del espacio turístico. México: Trillas.

Boyd, S., \& Singh, S. (2003). Destination communities: structures, resources and types. En S. Singh, D. Timothy, \& R. Dowling, Tourism in destination communities (págs. 19-34). Londres: CABI Publishing.

Bryman, A. (2012). Social research methods. Oxford: Oxford University Press. Foruth edition.

Cardenas, F. (2006). Proyectos turísticos: localización e inversión. México: Trillas.

Cardet, E., Palao, R., \& González, Y. (2018). Procedimiento para el diseño de productos turísticos basados en el patrimonio de un municipio. Retos de la dirección, 12(1), 1-16.

Castillo, E. (2015). Metodología para desarrollar productos turísticos. Caso de estudio. Red Internacional de Investigadores en Competitividad, 9(1), 97-108.

Consejo Nacional de Cultura y las Artes y Patrimonia Consultores. (2011). Portal del Departamento de Patrimonio Cultural inmaterial del Ministerio de las Culturas, Artes y el Patrimonio. Recuperado el 2018 de Diciembre de 12, de https://www.cultura.gob.cl/wp-content/uploads/2015/01/ guia-metodologica-turismo-cultural.pdf

Crespi, M., \& Planells, M. (2006). Productos y destinos tirísticos nacionales e internacionales. Madrid: Síntesis.

De Borja, L., Casanovas, J., \& Bosch, R. (2002). El consumidor turístico. Madrid: Esic Editorial.

Ernawati, N., Sudarmini, N., \& Sukmawati, N. (2018). Impacts of tourism in Ubud Bali Indonesia: a community based tourism perspective. Journal of Physics Conference Series, 953, 1-9. https://doi.org/10.1088/1742-6596/953/1/012078

Fernández, M. (2011). Turismo comunitario y empresas de base comunitaria turísticas: ¿Estamos hablando de lo mismo? EL Periplo Sustentable(20), 31-74.

Fondo Nacional de Turismo. (2014). Fondo Nacional de Turismo Colombia. Recuperado el 2018 de Noviembre de 20, de https://fontur.com.co/aym_document/aym_estudios_fontur/DISE- 


\section{NO_DE_PRODCTO_DE_HONDA.PDF}

Fundación CODESPA. (2011). Modelo de gestión del turismo rural comunitario de CODESPA. Una experiencia regional Andina. Lima: Edición CODESPA.

García, N., \& Quintero, Y. (2018). Producto de sol y playa para el desarrollo turístico del Municipio Trinidad de Cuba. Revista Interamericana de Ambiente y Turismo, 14(1), 52-64. http://dx.doi. org/10.4067/riat.v14i1.441

García, T., Figueiró, A., \& Silva, L. (2013). Possibilidades para um turismo de base comunitária na bacia do Rio Camaquã (RS): um diálogo com o desenvolvimento baseado no território. Revista Brasileira de Ecoturismo, 6(1), 139-152.

Go, F., \& Govers, R. (2000). Integrated quality management for tourist destinations: A European perspective on achieving competitiveness. Tourism Management, 21(1), 79-88. https://doi. org/10.1016/S0261-5177(99)00098-9

Godfrey, K., \& Clarke, J. (2000). The tourism development. Handbook, a practical approach to planning and marketing. London: Continuum.

Gómez, G. (2014). Procedimiento metodológico de diseño de productos turísticos para facilitar nuevos emprendimientos. Revista Retos, 8(1), 157-172. https://doi.org/10.17163/ret. n8.2014.08

Goodwin, H., \& Santilli, R. (2009). Community-Based Tourism: a success? ICRT y GTZ. Obtenido de http://www.haroldgoodwin.info/uploads/CBTaSuccessPubpdf.pdf

Gunn, C. (2002). Tourism Planing. United Kingdom: Routledge.

Hernández, A. (2001). Proyectos turísticos: formulación y evaluación. México: Trillas.

Hiwasaki, L. (2006). Community-Based Tourism: A pathway to sustainability for Japan's protected areas. Society and Natural Resources, 19(8), 675-692. https://doi. org/10.1080/08941920600801090

Ibañez, R., Ivanova, A., \& Amador, L. (2010). Turismo, comunidad y sustentabilidad en Cabo, Pulmo, Baja California Sur. En R. Chávez, E. Andrade, R. Espinoza, \& M. Navarro, Turismo comunitario en México. distintas visiones ante problemas comunes (págs. 35-50). México: Universidad de Guadalajara.

Ivars, J. (2003). Planificación turística. España: Síntesis.

Jaime, V., Casas, C., \& Soler, A. (2011). Desarrollo rural a través del turismo comunitario. Análisis del Valle y Cañón de Colca. Gestión turística, Enero-Junio(15), 1-20.

Jamal, T., \& Getz, D. (1995). Collaboration theory and community tourism planning. Annals of Tourism Research, 22(1), 186-204. https://doi.org/10.1016/0160-7383(94)00067-3

Jovicic, D. (2014). Key issues in the implementation of sustainable tourism. Current Issues in Tourism, 17(4), 297-302. https://doi.org/10.1080/13683500.2013.797386

Kotler, P., Haider, D., \& Rein, I. (1993). Marketing places: Attracting Investment, Industry, and Tourism to Cities, States and Nations. New York: The Free Press.

López-Guzmán, T., \& Sánchez, S. (2009). Turismo comunitario y generación de riqueza en países en vías de desarrollo. Un estudio de caso en El Salvador. REVESCO. Revista de Estudios Cooperativos(99), 85-103.

Luna, B., \& Polo, A. (2009). Elaboración de un producto turístico comunitario en las comunidades Puctahua y Llangahua del Cantón de Ambato, Provincia de Tungurahua. Tesis de ingenieria. Escuela Superior Politécnica de Chimborazo: Facultad de Recursos Naturales. Escuela de Ingenieria en Ecoturismo. Ecuador.

Luna, M. (2014). La participación de la comunidad local del municipio de Suesca en el desarrollo turístico, Colombia. Revista Interamericana de Ambiente y Turismo, 10(1), 33-39. http://dx.doi. org/10.4067/riatvol10iss1pp33-39\%250718-235X

Machado, E. (2013). Integración y diseño del producto turístico aplicado a la región central del destino Cuba. Revista Geographos, 4(35), 69-92. https://doi.org/10.14198/GEOGRA2013.4.35

Machado, E., \& Hernández, Y. (2007). Procedimiento para el diseño de un producto turistico integrado en Cuba. Teoria y Praxis(4), 161-174. 
Mair, H. (2012). The challenge of critical approaches to rural tourism studies and practice. En I. Ateljevic, N. Morgan, \& A. Pritchard, The critical turn in tourism studies (págs. 42-54). USA: Routledge.

Mehedi, M., Mohammed, A., Nassani, A., \& Nurul, M. (2017). Community-based ecoturism management for sustainable development of marine protected areas in Malaysia. Ocean $\mathbb{E}$ Coastal Management, 136, 104-112. https://doi.org/10.1016/j.ocecoaman.2016.11.023

Mikery, M., \& Pérez-Vázquez, A. (2014). Métodos para el análisis del potencial turístico del territorio rural. Revista Mexicana de Ciencias Agrícolas(9), 1729-1740.

Monterrubio, J. (2009). Comunidad receptora: elemento esencial en la gestión turística. Gestión Turística, 11, 101-11.

Montoya, M. (2013). Turismo comunitario y desarrollo rural. Interacción y escalonamiento de innovaciones. Lima: IEP, IDRC-CRDI; FIDA.

Monyara, G., \& Jones, E. (2007). Community-based tourism enterprises development in Kenya: an exploration of their potential as avenues of poverty reduction. Journal of Sustainable Tourism, 15(6), 628-644. https://doi.org/10.2167/jost723.0

Noia, A., Avila, M., \& Cartibani, M. (2009). Desarrollo turístico y comunidad local valoraciones y expectativas de los residentes de Ilhéus-BA, Brasil. Estudios y Perspectivas en turismo, 634-654.

Nyaupane, G., Morais, D., \& Dowler, L. (2006). The role of community involvement and number/ type of visitors on tourism impacts: A controlled comparison of Annapurna, Nepal and Northwest Yunnan, China. Tourism Management, 27(6), 1373-1385. https://doi.org/10.1016/j. tourman.2005.12.013

Okazaki, E. (2008). A community-based tourism model: Its conception and use. Journal of Sustainable Tourism, 16(5), 511- 529. https://doi.org/10.2167/jost782.0

Organización Mundial del Turismo. (1999). Agenda para planificadores locales: desarrollo turístico sostenible. España: OMT.

Osorio, M. (2006). La planificación turística, enfoques y modelos. Quivera. Revista de estudios urbanos, regionales, territoriales, ambientales y sociales, 8, 291-314.

Palomino, B., Gasca, J., \& López, G. (2016). El turismo comunitario en la Sierra Norte de Oaxaca: perspectiva desde las instituciones y la gobernanza en territorios indígenas. El Periplo Sustentable(30).

Paul, A. (2016). Propuesta de diseño de un producto turístico, para la comunidad Shuar de Tarimiat, Cantón Pasta$z a$, Provincia de Pastaza. Tesis de técnico en operación y comercialización. Quito: Universidad de las Américas. Facultad de turismo y hospitalidad.

Pearce, D. (1992). Alternative tourism: concepts, classifications and questions. En V. Smith, \& W. Eadington, Tourism alternatives: Potentials and problems in the development of tourism (págs. 15-30). Philadelphia: University of Pennsylvania Press.

Peterson, E., \& Barron, K. (2007). How to get focus group talking: new ideas that will Stick. International Journal of Qualitative Methods, 6(3), 140-144. https://doi.or$\mathrm{g} / 10.1177 \% 2 \mathrm{~F} 160940690700600303$

Programa de Cooperación al Desarrollo Económico. Secretaría de Estado para Asuntos Económicos SECO. Embajada Suiza en Perú. (2014). Manual para la planificación de productors turísticos. Lima: Swisscontact, Fundación Suiza de Cooperación para el Desarrollo Técnico.

Reyes, M., Ortega, Á., \& Machado, E. (2017). Modelo para la gestión integrada del turismo comunitario en Ecuador, caso de estudio de Pastaza. REVESCO. Revista de Estudios Cooperati$\operatorname{vos}(123), 250-275$.

Rodrigues, D., Corbari, S., Cioce, C., \& Juremma, I. (2014). Turismo comunitario en favelas. Un estudio del Favela Inn Hostel, Chapéu Mangueira - Rio de Janeiro, Brasil. Estudios y Perspectivas en Turismo, 23, 786-804.

Ruhanen, L. (2009). Steakeholder participation in tourism destination planning another case of missing the point? Tourism recreation research, 34(3), 283-294. https://doi.org/10.1080/02508 281.2009.11081603 
Ruiz, E., Hernández, M., Coca, A., Cantero, P., \& Del Campo, A. (2008). Turismo comunitario en Ecuador. Comprendiendo el community-based tourism desde la comunidad. PASOS. Revista de Turismo y Patrimonio Cultural, 6(3), 399-418.

Sampaio, C., Alves, F., \& Falk, V. (2008). Arranjo socioprodutivo de base comunitária: interconectando o turismo comunitario com redes de comércio justo. Turismo Visão e Ação, 10(2), 244-262.

Sánchez, L. (2009). Turismo y comunidad receptora. Un estudio en la costa venezolana. Fermentum, 19(54), 79-101.

Saravia, M., \& Muro, M. (2016). Productos turísticos. Metodología para su elaboración. Revista de Ciencias sociales, Segunda Época, 8(29), 53-78.

Sebele, L. (2010). Community-based tourism ventures, benefits and challenges: Khama Rhino Sanctuary Trust, Central District, Botswana. Tourism Management, 31(1), 136-146. https:// doi.org/10.1016/j.tourman.2009.01.005

Senyana, L., \& Moren, T. (2011). Community-based tourism enterprises: challenges and prospects for community participation; Khama Rhino Sanctuary Trust, Botswana. Journal of Sustainable Tourism, 19(1), 97-114. http://dx.doi.org/10.1080/09669582.2010.508527

Servicio Nacional de Turismo. (2015). Manual paso a paso para el diseño de productos turísticos integrados. Recuperado el 6 de Septiembre de 2018, de https://asesoresenturismoperu.files.wordpress.com/2016/08/244-manual-productos-turc3adsticos-chile.pdf

Singh, S., Timothy, D., \& Dowling, R. (2003). Tourism and Destination Communities. En S. Singh, D. Timothy, \& R. Dowling, Tourism in Destination Communities (págs. 3-18). Londres: CABI Publishing.

Teye, V., Sönmez, S., \& Sirakaya, E. (2002). Residents' attitudes toward tourism development. Annals of Tourism Research, 29(3), 668-688. https://doi.org/10.1016/S0160-7383(01)00074-3

Torres, J. (2015). Turismo comunitario y su estudio: Reflexiones desde un enfoque cultural propositivo para el desarrollo rural. Revista Humanismo y Cambio Social(5), 68-73. https://doi. org/10.5377/hcs.v0i5.4995

Ugarte, X. (2007). Imagen y posicionamiento de Galicia como destino turístico a nivel nacional e internacional. Galicia: Tesis Doctoral. Universidad de Santiago de Compostela.

Villalva, D. (2011). Diseños de productos turísticos sostenibles para el Cantón Cumandá provincia de Chimborazo. Tesis de ingenieria en Ecoturismo. Escuela Superior Politécnica de Chimborazo: Facultad de Recursos Naturales. Ecuador.

Waligo, V., Clarke, J., \& Hawkins, R. (2013). Implementing sustaiunable tourism: A multi-steakeholder involvement management framework. Tourism Management, 36, 342-353. https://doi. org/10.1016/j.tourman.2012.10.008

Zamorano, F. (2002). Turismo alternativo. México: Trillas. 